\title{
What lessons have been learnt from animal models of MRSA in the lung?
}

\author{
P. Martínez-Olondris*", M. Rigol*,*, and A. Torres*
}

ABSTRACT: Staphylococcus aureus is one of the most common causes of nosocomial pneumonia contributing to significant morbidity and mortality. Therapeutic options for patients with methicillin-resistant S. aureus (MRSA) infection are limited. In addition, little is known about the $S$. aureus virulence factors that may influence the presentation and prognosis of severe lower respiratory tract infections. Animal models of severe pneumonia allow investigators to control and exclude potential confounders and to examine the influence of comorbid conditions. Therefore, these models may improve our knowledge of the intimate pathophysiological mechanisms affecting pharmacodynamics, pharmacokinetics and efficacy of therapy. So far, animal research studies on MRSA and vancomycin-resistant $S$. aureus, performed both in small and large animal models, have improved knowledge of the mechanisms of disease, which may lead to a better treatment for this severe and complex infection in humans.

KEYWORDS: Animal model, lung, methicillin-resistant Staphylococcus aureus

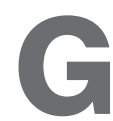
ram-positive bacteria have emerged as an important cause of hospital-acquired and community-acquired infections [1]. Staphylococcus aureus, in particular, is one of the most common causes of nosocomial pneumonia contributing to significant morbidity and mortality [2, 3]. An increasing prevalence (from $2 \%$ in 1974 to as high as $64 \%$ in recent surveys) of methicillinresistant S. aureus (MRSA) among nosocomial isolates has been identified [4-6]. Communityacquired MRSA is also becoming an important public health problem [7]. Although MRSA infection develops mainly in patients with risk factors related to healthcare institutions, it has also been described in the general population [8]. Therapeutic options for patients with MRSA infections are limited. Glycopeptides, such as vancomycin (VCM) and teicoplanin, were the most reliable therapeutic agents against this microorganism. However, since the first report of a Japanese patient harbouring a MRSA strain resistant to VCM in 1996 [9], isolation of reduced VCM susceptibility (SA-RVS) strains has been described in different countries such as the USA, France, Korea, South Africa and Brazil, confirming that the emergence of strains of $S$. aureus with resistance to VCM is a global issue [10]. The design of new therapeutic agents with activity in the face of this microorganism has been developed over recent years. Animal models have contributed significantly to this development and are an essential step between in vitro testing and human studies [11]. The availability of an animal model of are excluded or controlled may improve our knowledge on the intimal mechanisms involving the pathophysiology, pharmacology and efficacy of therapy. Here, we describe lessons that have been learnt regarding MRSA infection using animal models (table 1).

\section{SMALL ANIMAL MODELS OF MRSA- INDUCED PNEUMONIA}

There have been few experiments on small animal have been mainly carried out using mice and rats because they are cost-effective, easy to manage and genetically malleable. These recent studies have established the use of different routes of $S$. aureus inoculation to induce pneumonia as feasible and reproducible, including the intravenous and intrapulmonary pathways. severe pneumonia where potential confounders models of MRSA-induced pneumonia, and they

Previous article in this series: No. 1: Pantosti A, Venditti M. What is MRSA? Eur Respir J 2009; 34: 1190-1196. No. 2: Defres S, Marwick C, Nathwani D. MRSA as a cause of lung infection including airway infection, community-acquired pneumonia and hospital-acquired pneumonia. Eur Respir J 2009; 34: $1470-1476$.

\section{AFFILIATIONS}

*Servei de Pneumologia, Institut Clínic del Tòrax, Hospital Clínic, IDIBAPS (Institut d'Investigacions Biomèdiques Agustí Pi Sunyer), CIBERES (CIBER de Enfermedades Respiratorias) (06/06/0028),

Universidad de Barcelona, Barcelona, \#Servei de Cardiologia, Institut Clínic del Tòrax, Hospital Clínic, Red REDINSCOR RD06/0003/1002,

Barcelona, Spain.

"Both authors contributed equally to this work.

CORRESPONDENCE

A. Torres

Servei de Pneumologia

Institut Clínic del Tòrax

Hospital Clínic de Barcelona

Villarroel

170.08036 Barcelona

Spain

E-mail: atorres@clinic.ub.es

Received:

July 252008

Accepted after revision:

April 202009 
In 1997, SAWAI et al. [12] described a novel murine model of acute staphylococcal pneumonia induced by intravenous injection of $S$. aureus enmeshed in agar beads. This animal model is simple and reproducible and resembles blood-borne staphylococcal pneumonia in humans, and it could be useful for investigating the pathogenicity or treatment of staphylococcal pulmonary infection. When $S$. aureus was injected intravenously, the organisms concentrated and remained in the lungs for several weeks. Multiple lung abscesses were evident macroscopically, and histological examination of the infected lung showed multiple lung abscesses around the pulmonary arterioles, consisting of bacterial colonies encircled with fibrin filaments and surrounded by inflammatory cells of neutrophils and macrophages. Using this model, the authors studied the pathogenic role of staphylocoagulase, which is considered to be one of the most reliable determinants for differentiation of this bacterial species from other staphylococci, and also a critical virulence factor. SAWAI et al. [12] showed that the titre of staphylocoagulase correlated with the number of viable bacteria isolated from the lung on day 7 after inoculation. By contrast, injection of coagulase-deficient mutant strain was associated with a markedly reduced number of viable bacteria isolated from the lung. These results suggest that staphylocoagulase may play a role in the development of blood-borne staphylococcal pneumonia.

Using this model, the same group of investigators performed a series of studies evaluating the efficacy and pharmacokinetic profile of different agents with activity against MRSA. Their results demonstrated that, compared with VCM and teicoplanin, linezolid clearly reduces bacterial load in the MRSA haematogenous infection model and significantly improves survival rate. Interestingly, these authors reported, for the first time, evidence of in vivo efficacy of linezolid against SA-RVS infection. SA-RVS infection was reproduced by pre-treatment of animals with cyclophosphamide and then treating them with the test agent for 10 days using the same doses as those for MRSA. Treatment commenced 1 day after inoculation by intraperitoneal administration of the test agent. As with the MRSA experiments, animals suffered from SA-RVS infection and when treated with linezolid had a better pharmacokinetic profile, lower bacterial counts and a better survival rate than animals with the same infection treated with VCM or teicoplanin [13].

The identification of a MRSA strain resistant to linezolid from a patient with dialysis-associated peritonitis reinforced the need to develop new potent antimicrobial agents against SA-RVS [24]. KANEKO et al. [14], using the previously described animal model, evaluated the antibacterial and histopathological effects of DQ113, a new quinolone, with activity against MRSA and SA-RVS. They compared the new agent with VCM and teicoplanin. DQ113 significantly reduced the number of viable bacteria in the lung compared with VCM and teicoplanin. Histopathological examination revealed milder inflammatory changes in the group of quinolone-treated mice than in the other groups. The pharmacokinetics parameters, such as the area under the concentration-time curve from 0 to $6 \mathrm{~h}\left(\mathrm{AUC}_{0-6}\right) /$ minimum inhibitory concentration (MIC) ratio, or the time that the $\mathrm{AUC}_{0-6}$ exceeded the MIC, were the highest for DQ-113. These results suggest that DQ-113 is a potent and effective agent for the treatment of haematogenous pulmonary infections caused by MRSA and SA-RVS strains [14]. Also, this same group of investigators evaluated the effect of quinupristin-dalfopristrin (Q-D) (a complex of streptogramin A and B) and DX-619, a novel des-fluoro(6)-quinolone, with that of VCM against MRSA and SA-RVS. Treatment with Q-D and DX-619 resulted in a significant decrease in the number of viable bacteria in the lungs of mice in a MRSA infection model $[15,16]$.

Finally, the efficacy of short interfering RNA (siRNA) on the expression of coagulase, one of the most important enzymes in the pathogenesis of MRSA infection, was evaluated by YANAGIHARA et al. [17] using the previously described animal model of i.v. inoculation of $S$. aureus. They examined the

\section{TABLE 1 Animal models of methicillin-resistant Staphylococcus aureus lung infection}

\section{Small animal models}

Murine model of haematogenous pulmonary infection by intravenous injection of $S$. aureus enmeshed in agar beads

SAWAl et al. [12]: animal model validation and role of coagulase (February 1997)

YANAGIHARA et al. [13]: efficacy of linezolid against methicillin-resistant or vancomycin-insensitive S. aureus (July 2002)

KANEKO et al. [14]: effects of DQ-113 against methicillin- and vancomycin-resistant S. aureus (August 2003)

YANAGIHARA et al. [15]: efficacy of quinpristin-dalfopristin against methicillin-resistant S. aureus and vancomycin-insensitive S. aureus (November 2004)

YANAGIHARA et al. [16]: potency of DX-619 against methicillin-resistant and vancomycin-intermediate S. aureus (March 2006)

YANAGIHARA et al. [17]: effects of short interfering RNA against methicillin-resistant S. aureus coagulase in vitro and in vivo (December 2005)

Murine model of pulmonary infection by transnasal administration of $S$. aureus

KIMURA et al. [18]: animal model validation and factors affecting the course and severity of pneumonia (November 1999)

BUBECK WARDENBURG et al. [19]: surface proteins and exotoxins are required for the pathogenesis of S. aureus pneumonia (February 2007)

BUBECK WARDENBURG et al. [20]: vaccine protection against S. aureus pneumonia (February 2008)

Murine model of pulmonary infection by instillation into the lung of $S$. aureus in ventilated animals

MCELROY et al. [21]: alpha-toxin damages the air-blood barrier (October 1999)

Large animal models

Ovine ventilated model of pulmonary infection by instillation by bronchoscope of $S$. aureus

ENKHBAATAR et al. [22]: animal model validation (May 2008)

Piglet ventilated model of pneumonia induced by instillation by broncoscope of $S$. aureus

SIBILA et al. [23]: animal model validation (2007) 
inhibitory effect of siRNA on staphylocoagulase in vitro and in vivo. The results showed that siRNA inhibited both mRNA expression and the activity of MRSA coagulase in vitro. The in vivo results revealed that the siRNA was effective in reducing the bacterial load. Targeting coagulase with siRNA appears to be a novel and promising strategy for treating MRSA infections.

Regarding the studies on acute staphylococcal pneumonia induced by intranasal or intrapulmonary injection of S. aureus, in 1999 KIMURA et al. [18] examined several factors that may affect the course and severity of $S$. aureus pneumonia in five strains of immunocompromised mice. Immunosuppression was induced by injection of cyclophosphamide and impairment of mucociliary clearance by intranasal instillation of formalin. Authors found that pre-treatment with formalin plus cyclophosphamide was associated with a significant increase in lung bacterial counts and a high mortality. Moreover, CBA/J mice represented the most susceptible strain among those examined. Therefore, they demonstrated that neutropenia and impaired mucociliary clearance were major factors that influence the severity of pneumonia in mice. However, although this animal model emphasised the inflammatory response to intrapulmonary $S$. aureus, characterisation of bacterial virulence factors was not possible because of the quick clearance of $S$. aureus from the lungs. In contrast, BUBECK WARDENBURG et al. [19] more recently developed a transnasal murine model of $S$. aureus pneumonia in adult and immunocompetent C57BL/6J mice. They found that this model closely mimics the clinical and pathological features of pneumonia in human patients and allows investigation of virulence factors such as the pore-forming $\beta$-barrel toxin Hla. Later, these authors used this experimental model to examine Hla as a target for the development of vaccines that combat $S$. aureus lung infection [20]. They demonstrated that the relative level of Hla expression by distinct $S$. aureus strains correlated with the virulence properties of the organism. Moreover, they reported that active immunisation with a mutant form of Hla protected mice from staphylococcal pneumonia, and that this protection correlated with reduced microbiological and pathological evidence of disease. In addition, these authors passively immunised animals with Hla-specific antibodies and demonstrated that passive immunoprotection protected animals from S. aureus pneumonia, correlating with favourable alterations in the cytokine profile of the host. In agreement with these results, McELroy et al. [21] determined the role of alpha-toxin by developing a rat model of $S$. aureus-induced pneumonia. These authors instilled $S$. aureus strain 8325-4 into the lungs of ventilated Sprague-Dawley rats and demonstrated that the function of the air-blood barrier was impaired in S. aureusinduced pneumonia and that alpha-toxin was an important cause of damage to the air-blood barrier.

Recently, a study by REYES et al. [25] used a similar murine pneumonia model to compare the efficacy of telavancin with that of VCM and linezolid against MRSA. In particular, the authors inoculated $10^{7} \mathrm{CFU}$ of MRSA onto the tip of the nares of neutropenic mice, and $12 \mathrm{~h}$ and $24 \mathrm{~h}$ after inoculation the animals were dosed with telavancin, VCM or linezolid. They found that when dosed both at $12 \mathrm{~h}$ and at $24 \mathrm{~h}$ after inoculation, animals treated with telavancin had a significantly greater reduction in lung bacterial titre than those treated with VCM or linezolid. Moreover, when the animals were dosed at $24 \mathrm{~h}$ after inoculation, telavancin and VCM-treated groups showed significantly greater improvement in survival of mice than did controls and linezolidtreated groups, suggesting the potential utility of telavancin for treatment of MRSA pneumonia.

\section{LARGE ANIMAL MODELS OF MRSA-INDUCED PNEUMONIA}

Although small animals offer the advantages of low cost, genetic malleability and high throughput, they cannot reproduce many aspects of human pulmonary pathophysiology. For this reason, it is very important to use physiologically relevant large animal models whenever possible. However, if there have been few experimental studies on the development of small animal models of MRSA-induced pneumonia, there have been even fewer using large animal models of MRSA. Nevertheless, it is important to point out the relevance of the study of ENKHBAATAR et al. [22], using an ovine model of MRSA-induced pneumonia and sepsis that closely mimics hyperdynamic human sepsis. Authors established a standardised and reproducible model of MRSA-induced septic pneumonia by instillation of $S$. aureus, with and without smoke injury induced by inhalation of cotton smoke. They found that animals exposed to smoke inhalation and MRSA showed the signs of severe sepsis-related multiple organ failure $3 \mathrm{~h}$ after insult. Pulmonary dysfunction was characterised by deteriorated gas exchange and increased ventilatory pressure. Moreover, animals showed significantly greater lung tissue water, myeloperoxidase activity and cytokine production compared with uninjured animals. Conversely, using this novel ovine model, the authors found that all these changes were accompanied by an increase in plasma nitrite/nitrate and reactive nitrogen species in the lungs, suggesting that excessive nitric oxide production may be involved in the pathogenic process.

Another relevant study about the development of a large animal model of MRSA-induced pneumonia was carried out in pigs. SiBILA et al. [23] validated a porcine model of MRSA pneumonia and studied the time-course of biological markers and histopathological changes. The authors found that, in ventilated pigs, bronchoscopic inoculation of MRSA induced pneumonia at $12 \mathrm{~h}$ and severe pneumonia at $24 \mathrm{~h}$. This severity was associated with an increase in interleukin (IL)-6, IL-8 and tumour necrosis factor- $\alpha$ at $24 \mathrm{~h}$ after inoculation. Therefore, the authors showed that this experimental model in ventilated piglets closely mimics MRSA human pneumonia and it could lead to future studies on the effects of different antimicrobial therapies against MRSA pneumonia.

\section{CONCLUSION}

S. aureus is one of the most common causes of nosocomial pneumonia contributing to significant morbidity and mortality. Therapeutic options for patients with MRSA infection are limited. In addition, little is known about the $S$. aureus virulence factors that may influence the presentation and prognosis of severe lower respiratory tract infections. Animal models of severe pneumonia allow investigators to control and exclude potential confounders and to examine the influence of co-morbid conditions. Therefore, these models may improve our knowledge on the intimate pathophysiological mechanisms affecting pharmacodynamics, pharmacokinetics and efficacy of therapy. So far, animal research studies on MRSA 
and SA-RVS, performed both in small and large animal models, have provided further insight into the mechanisms of disease that may lead to a better treatment of this severe and complex infections in the human.

\section{SUPPORT STATEMENT}

This work was funded in part by the Ministerio de Ciencia e Innovación (Madrid, Spain), Instituto de Salud Carlos III (Red HERACLES RD06/0009, Red REDINSCOR RD06/0003/1002) (Madrid Spain), CIBERES (CIBER de Enfermedades Respiratorias) and Fundación Lilly.

\section{STATEMENT OF INTEREST}

None declared.

\section{REFERENCES}

1 Fluckiger U, Widmer AF. Epidemiology of methicillin-resistant Staphylococcus aureus. Chemotherapy 1999; 45: 121-134.

2 National Nosocomial Infections Surveillance (NNIS) System report, data summary from October 1986 through April 1998. Atlanta, Hospital Infections Program, National Center for Infectious Diseases, Centers for Disease Control and Prevention, 1998.

3 Kollef MH, Morrow LE, Niederman MS, et al. Clinical characteristics and treatment patterns among patients with ventilatorassociated pneumonia. Chest 2006; 129: 1210-1218.

4 Maranan MC, Moreira B, Boyle-Vavra S, et al. Antimicrobial resistance in staphylococci: epidemiology, molecular mechanisms, and clinical relevance. Infect Dis Clin North Am 1997; 11: 813-849.

5 Panlilio AL, Culver DH, Gaynes RP, et al. Methicillin-resistant Staphylococcus aureus in US hospitals, 1975-1991. Infect Control Hosp Epidemiol 1992; 13: 582-586.

6 Voss A, Milatovic D, Wallrauch-Schwarz C, et al. Methicillinresistant Staphylococcus aureus in Europe. Eur J Clin Microbiol Infect Dis 1994; 13: 50-55.

7 Fridkin SK, Hageman JC, Morrison M, et al. Methicillin-resistant Staphylococcus aureus disease in three communities. N Engl J Med 2005; 352: 1436-1444.

8 Centers for Disease Control and Prevention. Staphylococcus aureus resistant to vancomycin-United States, 2002. Mob Mortal Wkly Rep 2002; 51: 565-567.

9 Hiramatsu $\mathrm{K}$, Hanaki $\mathrm{H}$, Ino $\mathrm{T}$, et al. A methicillin-resistant Staphylococcus aureus clinical strain with reduced vancomycin susceptibility. J Antimicrob Chemother 1997; 40: 135-146.

10 Smith TL, Pearson ML, Wilcox KR, et al. Emergence of vancomycin resistance in Staphylococcus aureus. N Engl J Med 1990; 340: 493-501.

11 Rybak MJ, Hershberger E, Moldvan $\mathrm{T}$, et al. In vitro activities of daptomycin, vancomycin, linezolid, and quinupristindalfopristin against staphylococci and enterococci, including vancomycin-intermediate and -resistant strains. Antimicrob Agents Chemother 2000; 44: 1062-1066.

12 Sawai T, Tomono K, Yanagihara K, et al. Role of coagulase in a murine model of hematogenous pulmonary infection by intravenous injection of Staphylococcus aureus enmeshed in agar beads. Infect Immun 1997; 65: 466-471.

13 Yanagihara K, Kaneko Y, Sawai T, et al. Efficacy of linezolid against methicillin-resistant or vancomycin-insensitive Staphylococcus aureus in a model of hematogenous pulmonary infection. Antimicrob Agents Chemother 2002; 46: 3288-3291.

14 Kaneko Y, Yanagihara K, Miyazaki Y, et al. Effects of DQ-113, a new quinolone, against methicillin- and vancomycin-resistant Staphylococcus aureus-caused hematogenous pulmonary infections in mice. Antimicrob Agents Chemother 2003; 47: 3694-3698.

15 Yanagihara K, Okada M, Fukuda Y, et al. Efficacy of quinpristindalfopristin against methicillin-resistant Staphylococcus aureus and vancomycin-insensitive $S$. aureus in a model of hematogenuos pulmonary infection. Chemotherapy 2004; 50: 260-264.

16 Yanagihara K, Seki M, Izumikawa K, et al. Potency of DX-619, a novel des-F(6)-quinolone, in haematogenous murine bronchopneumonia caused by methicillin-resistant and vancomycinintermediate Staphylococcus aureus. International I Antimicrob Agents 2006; 28: 212-216.

17 Yanagihara K, Tashiro M, Fukuda Y, et al. Effects of short interfering RNA against methicillin-resistant Staphylococcus aureus coagulase in vitro and in vivo. J Antimicrob Chemotherapy 2006; 57: 122-126.

18 Kimura K, Miyazaki S, Tateda K, et al. Factors affecting the course and severity of transnasally induced Staphylococcus aureus pneumonia in mice. J Med Microbiol 1999; 48: 1005-1010.

19 Bubeck Wardenburg J, Patel RJ, Schneewind O. Surface proteins and exotoxins are required for the pathogenesis of Staphylococcus aureus pneumonia. Infect Immun 2007; 75: 1040-1044.

20 Bubeck Wardenburg J, Schneewind O. Vaccine protection against Staphylococcus aureus pneumonia. J Experimental Med 2008; 205 287-294.

21 McElroy MC, Harty HR, Hosford GE, et al. Alpha-toxin damages the air-blood barrier of the lung in a rat model of Staphylococcus aureus-induced pneumonia. Infect Immun 1999; 67: 5541-5544.

22 Enkhbaatar P, Joncam C, Traber L, et al. Novel ovine model of methicillin-resistant Staphylococcus aureus-induced pneumonia and sepsis. Shock 2008; 29: 642-649.

23 Sibila O, Martinez-Olondris $\mathrm{P}$, Agustí C, et al. An experimental model of pneumonia induced by MRSA in ventilated piglets. Am J Respir Crit Care Med 2007; 175: A327.

24 Tsiodras S, Gold HS, Sakoulas G, et al. Linezolid resistance in a clinical isolate of Staphylococcus aureus. Lancet 2001; 358: 207-208.

25 Reyes N, Skinner R, Benton BM, et al. Efficacy of telavancin in a murine model of bacteraemia induced by methicillin-resistant Staphylococcus aureus. J Antimicrob Chemother 2006; 58: 462-465. 